\title{
The Importance of Torque/Speed-Points and Working Cycles in Designing Electric Motors
}

\author{
Johannes Schmelcher \\ University of Applied Sciences Ravensburg-Weingarten, Weingarten, Germany \\ Max Kleine Büning \\ Chair of Production Engineering of E-Mobility Components PEM, \\ RWTH Aachen University, Aachen, Germany \\ Kai Kreisköther \\ Chair of Production Engineering of E-Mobility Components PEM, \\ RWTH Aachen University, Aachen, Germany \\ Dieter Gerling \\ University of Federal Defence Munich, Neubiberg, Germany \\ Achim Kampker \\ Chair of Production Engineering of E-Mobility Components PEM, \\ RWTH Aachen University, Aachen, Germany
}

\begin{abstract}
Designing electric motors is a task, where various requirements and objectives have to be met simultaneously. To achieve this, different approaches to the design optimization problem of electric motors have been developed. Especially today, one of the most important properties of an electric motor is its efficiency. Therefore, it is important to include the efficiency into the design process since at this stage, gains in energy efficiency can be made very cost-effective. Typically, state of the art is to consider only distinct operational points and not the whole operational cycle in the design optimization. To demonstrate that the whole working cycle of an electric motor should be included into the design optimization, is subject of this paper. Based on a literature survey of recent design optimization methods the current state of the art will be presented. The main objective of this paper is dedicated to the design of a permanent magnet synchronous motor. Using genetic algorithms, the motor will be designed several times with varying operational points. As will be seen, for the sake of minimal energy requirement, the whole working cycle and not only distinct operational points have to be considered in the design optimization.
\end{abstract}

Keywords: Electric motor, Design optimization, Genetic algorithm, Operational cycle, Efficiency, Energy, Energy-efficient motors 


\section{Introduction}

Electric motors are gathering increasing importance in recent years due to electric vehicles, their application in industry and in domestic appliances. This leads to an increasing share of electric motors in the world-wide energy consumption. In 2014, it was estimated that electric motors are responsible for $53 \%$ of the global electricity consumption (van Werkhoven \& Advani, 2017). If this fraction is divided into different sectors of applications, the industrial sector is the most important with a share of at least $69 \%$ of energy consumed by electric motors. The second most important sector is transportation with a share of $60 \%$ (Waide \& Brunner, 2011). To gain such a high popularity, electric motors have to fulfil various complex requirements. Therefore, various methodologies have been developed to design electric motors which meet all the requirements. Traditionally, these methodologies were manual processes where the different aspects of the electric motors were determined in a systematic fashion (Müller et al., 2008) (Pyrhönen et al., 2010). More recent approaches use numeric methods in conjunction with optimization methods to obtain the correct values for a motor design (Zhu et al., 2017) (Wu et al., 2015) (Khelifa et al., 2017). Probably the most important criterion for a motor design proposal is its efficiency. The efficiency is hugely dependent on the operating conditions. Typically, in the designing process of an electric motor, some distinct operating points are used. These points can be the effective torque and the average speed, the maximum torque and the corresponding speed, or some other important point. However, an electric motor has to cope with several different operating conditions which might not be considered in the design stage.

The paper is divided into five sections. After this introductory section, recent design optimization methods are shortly summarized. It will become apparent, how the different methods deal with the working cycle of the electric motor and what the objectives are. To illustrate what consequences the choice of either different operational points or the whole working cycle have concerning the efficiency respectively the required energy, multiple design optimization are carried out exemplarily. For this purpose, requirements which the permanent magnet synchronous motor (PMSM) has to fulfil are presented in section three. After that, the workflow and the software used are shortly summarized in section four. The main part will be section five, in which the results of the design optimizations will be stated and compared. The closing section of this paper is dedicated to a summary of the findings and concluding remarks. 


\section{State of the Art}

State of the art in the design optimization of electric motors is to define a characteristic operational point, which is the foundation for the forthcoming optimization. Typically, the design points are based on peak as well as rated power and torque values. Other approaches use exiting motor design to derive the required design points and use these as the inputs to the design optimization. Approaches, which use these kinds of definitions for the inputs have in common, that the operational cycle is not present in the optimization process. The objectives, like for instance the efficiency, the torque or the active material costs or multi-objective combinations of them, are determined in the design points. Exemplarily for these approaches Jung et al. (2018), Zuopeng et al. (2017) and Haifeng et al. (2017) can be named here.

Some recent approaches try to give the working cycle more importance in the optimization process. In $\mathrm{Li}$ et al. (2017) the operational cycle is divided into a number of squares, for each of which the equivalent torque and speed are computed as the geometrical center of gravity. These points are weighted according to the importance of the respective square concerning the whole operational cycle. In the subsequent optimization process, these equivalent working points are used as the input. Another approach is used by Fatemi et al. (2016), where a method from data mining is used to cluster the operational cycle. The k-means clustering algorithm iteratively groups the operational points into a finite number of clusters. Each cluster is assigned a representative torque and speed as well as a weighting factor to account for the energy consumed in the respective cluster. These representative cluster points are used in the optimization and not the whole working cycle. The objectives of the optimization process are comparable to the previously mentioned ones. For all cluster points, the objectives are computed and averaged with respect to weighting factors.

This short literature survey of recent design optimization methods reveals that very few methods consider the operational cycle at all. Most approaches design the electric motor with respect to one or more design points. These points typically represent an important operational condition. Approaches, where the operational cycle is included in the optimization, condense them into a small number of equivalent design points. In these design points, the objectives are computed, averaged and used in the optimization process.

Seemingly no approaches are apparent, where the working cycle as a whole was included in the design optimization process nor that the objective is the energy required to conduct the working cycle. In the following it will be shown how important it is to use the whole 
operational cycle as the input to the optimization process and the required energy as the objective in order to design an electric motor.

\section{Requirements}

The task of designing an electric motor, in this case a PMSM, is challenging because of the numerous influential parameters. In the following, the different fixed and variable design parameters are detailed. These requirements are based on a real use case, in which a motor for an electric vehicle has to be designed.

\subsection{Working Cycle}

The working cycle acts like a boundary condition. Every valid design proposal must be able to conduct the working cycle. Based on this cycle, different design points can be derived, which will be presented in more detail in the following section. In this particular case, the working cycle is a driving cycle for an electric scooter. The maximum speed is $45 \mathrm{~km} / \mathrm{h}$ and the scooter has a total weight of $100 \mathrm{~kg}$. Finally, the wheels have a diameter of 12 inches. With the help of a physical model, the driving cycle of the scooter can be turned into a torque-speed diagram or working cycle for the PMSM, which can be seen in Figure 1.

Figure 1. Driving cycle of an electric scooter and working cycle of a PMSM. The numbered dots indicate the respective design points.
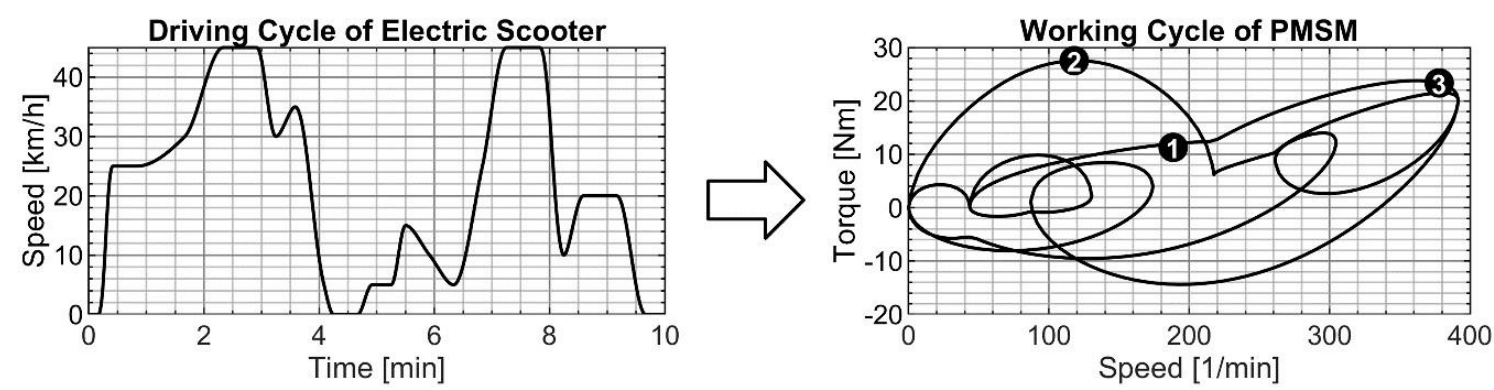

\subsection{Design Points}

Based on the working cycle, different torque/speed design points can be defined. In this paper, three different design points are chosen in addition to the whole working cycle.

1. $T_{\text {eff }}=11,3 \mathrm{Nm}$ and $n_{\text {avg }}=188,51 / \mathrm{min}$ : These effective quantities are the result of converting the time varying torque and speed into constant values, which will produce an equivalent load for the PMSM. 
2. $T_{\max }=27,6 \mathrm{Nm}$ and corresponding $n=1181 / \mathrm{min}$ : Since the maximum torque of the working cycle is considered here, the maximum current will be necessary, leading to great ohmic losses.

3. $\quad T=23,4 \mathrm{Nm}$ and $n=3781 / \mathrm{min}$ at $P_{\max }$ : Maximum power is a combination of torque and speed, therefore considerable amounts of ohmic and core losses have to be dealt with.

The choice of proper design points is not a trivial task and has great impact on the design optimization process. Therefore, the design points should be chosen carefully. In addition to the aforementioned design points, a fourth design optimization is carried out where the whole working cycle has to be regarded.

\subsection{Geometric Requirements}

In the design optimization process, various geometrical requirements have to be fulfilled. The PMSM has to be designed as an outside rotor motor with surface mounted permanent magnets and three phases. In order to allow a comprehensive design optimization, most dimensions of the PMSM are not restricted. The only restrictions which have to be respected are the outer dimensions of the motor, the minimum inner diameter of the stator as well as the slot and pole number. Concerning the outer dimensions, the diameter and the thickness are not allowed to exceed $350 \mathrm{~mm}$ respectively $50 \mathrm{~mm}$. For the inner diameter of the stator, the lower limit is $50 \mathrm{~mm}$. Additionally, valid values for the air gap width are between 0,5 $\mathrm{mm}$ and $2 \mathrm{~mm}$. The slot number is chosen to be 48 and the pole number to be 44 . All the other dimensions can be chosen freely, as long as the resulting geometry of the PMSM is valid. This means that no geometrical elements are overlapping or intersecting.

Figure 2. Theoretical minimum and maximum valid motor geometries. The illustrations are not in scale.
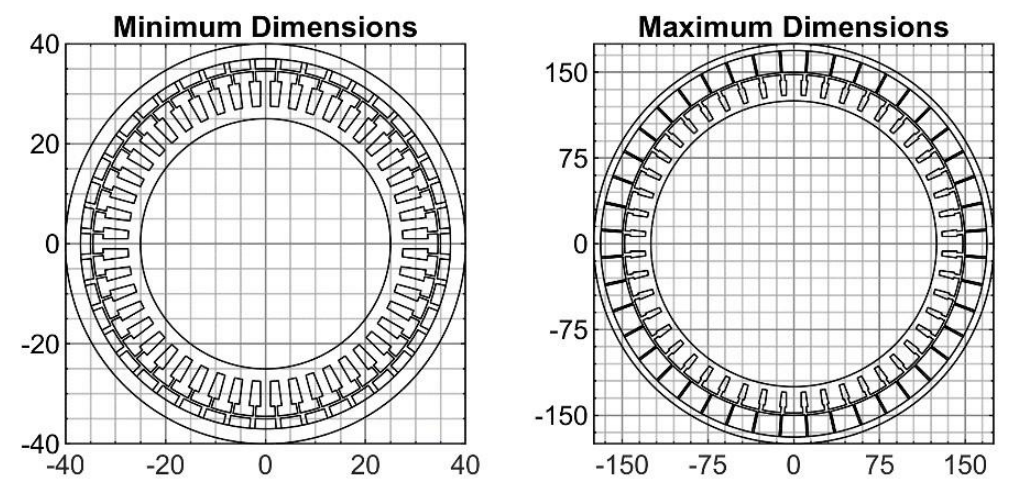

\subsection{Electrical Requirements}

The electric scooter is battery-powered with a nominal voltage of $48 \mathrm{~V}$. To ensure 
the thermic integrity of the coils and to prevent short-circuits due to overheating of the coils, a maximum current density of $5 \mathrm{~A} / \mathrm{mm}^{2}$ is admissible. The coils consist of wires with a maximum diameter of $1 \mathrm{~mm}$. If higher cross sections of copper wire are required, up to 5 strands can be wound in parallel. The coils are realized as double layered concentrated windings.

\subsection{Materials}

The material selection for the PMSM is not part of this design optimization. For the stator, the electric steel sheet M250-35A with a thickness of 0,35 $\mathrm{mm}$ and losses of 2,35 $\mathrm{W} / \mathrm{kg}$ at 1,5 $\mathrm{T}$ and $50 \mathrm{~Hz}$ is used. Since core losses in the rotor are not very prominent, the material selected for the rotor is the general-purpose low carbon steel C15E. The permanent magnets used are neodymium iron boron magnets $(\mathrm{NdFeB})$. To keep the required magnet volume low, a magnet type which has a high energy product is used. Since demagnetization due to high temperatures is a risk, an appropriate magnet type has to be chosen. This leads to the selection of the permanent magnet with the grade $\mathrm{N} 42 \mathrm{H}$.

Table 1. Requirements for the design optimization.

\begin{tabular}{cccc}
\hline Parameter & Value & Parameter & Value \\
\hline Minimum Inner Stator Diameter & $50 \mathrm{~mm}$ & Number of Slots & 48 \\
Maximum Outer Rotor Diameter & $350 \mathrm{~mm}$ & Number of Poles & 44 \\
Maximum Motor Thickness & $50 \mathrm{~mm}$ & Nominal Voltage & $48 \mathrm{~V}$ \\
Air Gap Width & $0,5 \ldots 2 \mathrm{~mm}$ & Maximum Current Density & $5 \mathrm{~A} / \mathrm{mm}^{2}$ \\
Material Stator & $\mathrm{M} 250-35 \mathrm{~A}$ & Maximum Wire Diameter & $1 \mathrm{~mm}$ \\
Material Rotor & $\mathrm{C} 15 \mathrm{E}$ & Maximum Parallel Strands & 5 \\
Material Magnets & $\mathrm{N} 42 \mathrm{H}$ & & \\
\hline
\end{tabular}

\section{Design Optimization Process}

The design optimization process requires a planned and systematic approach to determine the optimal motor topology. Since the design optimization method is not the scope of this paper, a proven method similar to Zhu et at. (2017) has been used. In the following the utilized software and the workflow are presented shortly.

\subsection{Software}

Two different kinds of software are used for the design optimization process. The first is MATLAB with its built-in genetic algorithm capabilities, which are used for the gradient-free optimization of the PMSM. This algorithm offers the possibility to define bounds as well as nonlinear constraints for the design variables and is well proven in 
many different research areas. The other software used is xfemm from Crozier and Mueller (2016). It was branched off Finite Element Method Magnetics from Meeker (2018) and offers similar capabilities, with the benefit of APIs for MATLAB.

\subsection{Workflow}

The workflow of the design optimization can basically be divided into an outer and an inner process. The outer process is dedicated to the genetic algorithm, whereas the inner process deals with the calculation of the efficiency respectively the energy. In Figure 3, the general workflow can be seen.

Figure 3. Schematic workflow of the design optimization process.

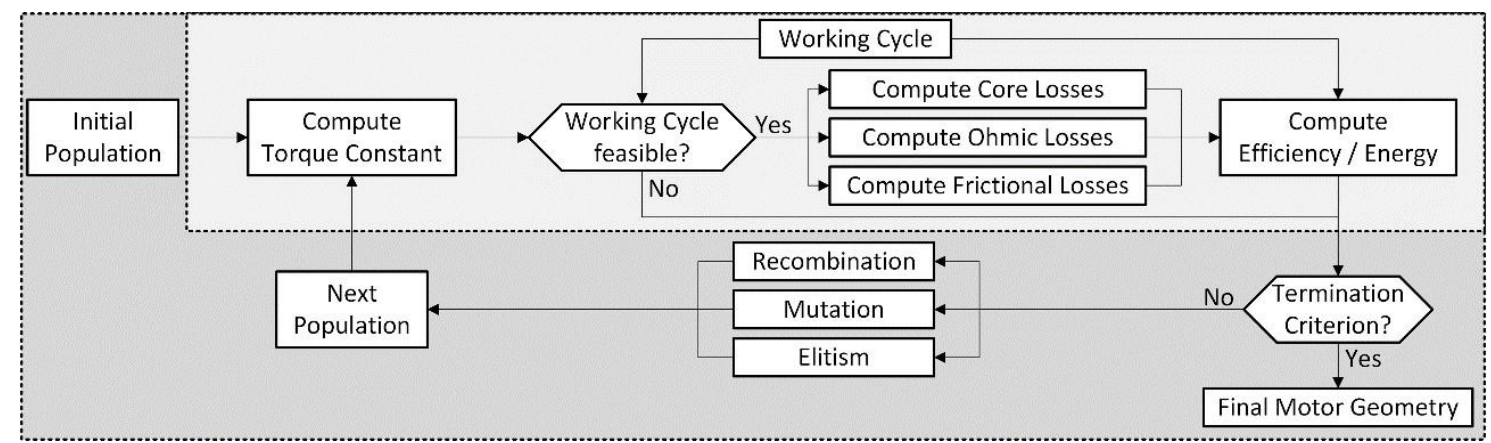

The characteristics of the genetic algorithm are as follows. The fitness function, which characterizes the different individuals and expresses if they are better or worse than others, is the efficiency at the design point for the first three design optimizations. For the fourth optimization, the whole working cycle of the PMSM is considered and therefore the energy required to conduct this cycle is the fitness function. The goal is to maximize the efficiency and minimize the energy. Each population consists of 50 individuals, where the initial population is the same for each of the four design optimizations. To create the upcoming generation, $70 \%$ of the new generation is formed by recombination, $25 \%$ are individuals which undergo mutation and the final $5 \%$ are the elite individuals, which pass to the next generation unchanged. Since the fitness functions are rather time-consuming to calculate, the fraction of mutated individuals is chosen purposely high. This will compensate for the termination criterion of 50 generations while still allowing a thorough exploration of the design space.

The first step in the inner process of calculating the fitness function is to check if the individuals are able to perform the whole working cycle of the PMSM. For this purpose, the torque constants are computed with the help of a custom-written MATLAB function and xfemm. Then it is checked if all the working points of the PMSM are feasible for each individual. This is dependent on the torque constants, the speed and thus the 
available current. For individuals, which are not able to perform the working cycle, the further computations are skipped. For the other ones, the different losses are calculated at the design points or for the whole working cycle. The core losses are computed via a time-stepped finite element analysis using xfemm and Fourier analysis. Ohmic losses are calculated analytically based on the dimensions of the motor proposal and the required current. The friction losses are estimated according to empirical formulas. These calculations concerning the different losses are performed in individual MATLAB functions. With these losses and the requested output power according to the working cycle, the efficiency respectively the energy can be determined. Thus, the fitness function for each individual is calculated and the next generation can be created.

\section{Results}

After completing the design optimization process with respect to the individual design points respectively the whole working cycle, the resulting design proposals are presented, evaluated and compared to each other.

\subsection{Design Point 1: Teff $_{\text {and }} \boldsymbol{n}_{\text {avg }}$}

The design optimization process results in a proposal regarding the geometry of the PMSM which is depicted in the middle of Figure 4. Additionally, the course of the efficiency over the elapsed generations as well as the resulting efficiency map can be seen.

Figure 4. Results of the design optimization process with respect to design point 1, indicated as a black dot in the efficiency map on the right.
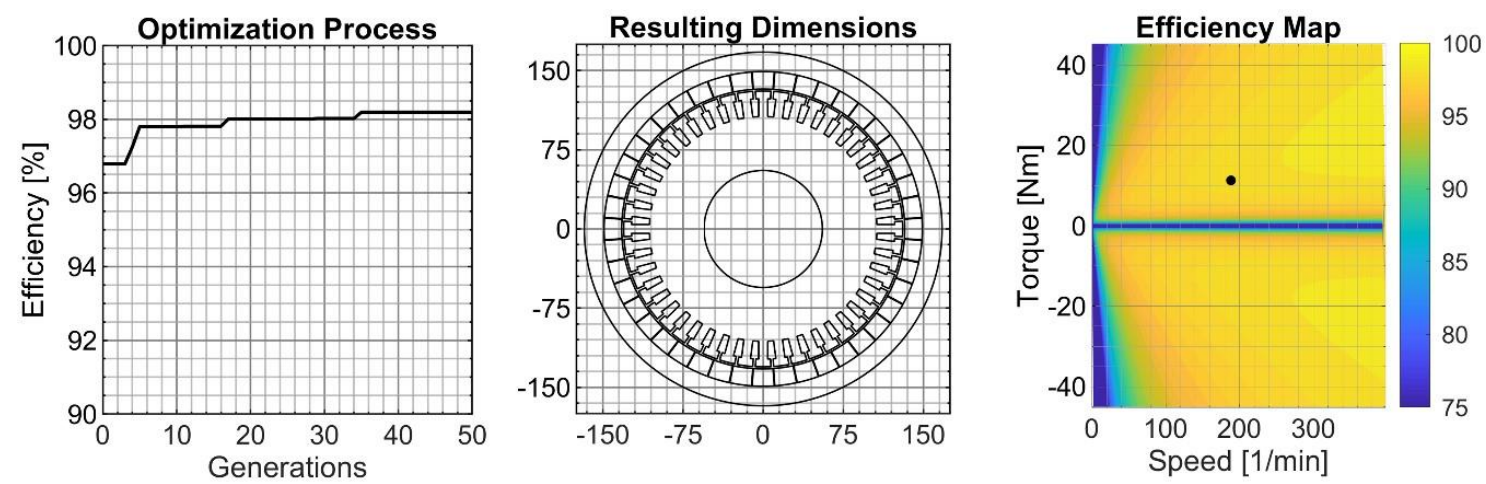

The final design is characterized by an outer diameter of $334,9 \mathrm{~mm}$, a thickness of $17,85 \mathrm{~mm}$, an air gap width of $1,9 \mathrm{~mm}$. In this design point, the efficiency is $98,3 \%$ and the motor has to generate a power output of $223 \mathrm{~W}$. The rated speed is $396,11 / \mathrm{min}$ and the rated torque $22,6 \mathrm{Nm}$, leading to a nominal power of $938,4 \mathrm{~W}$. 


\subsection{Design Point 2: $\boldsymbol{T}_{\max }$ and corresponding $n$}

For the design optimization of the following design proposal, not the effective torque and average speed is used, but the maximum required torque and the corresponding speed. In Figure 5, the geometry of the PMSM can be seen in the middle.

Figure 5. Results of the design optimization process with respect to design point 2, indicated as a black dot in the efficiency map on the right.
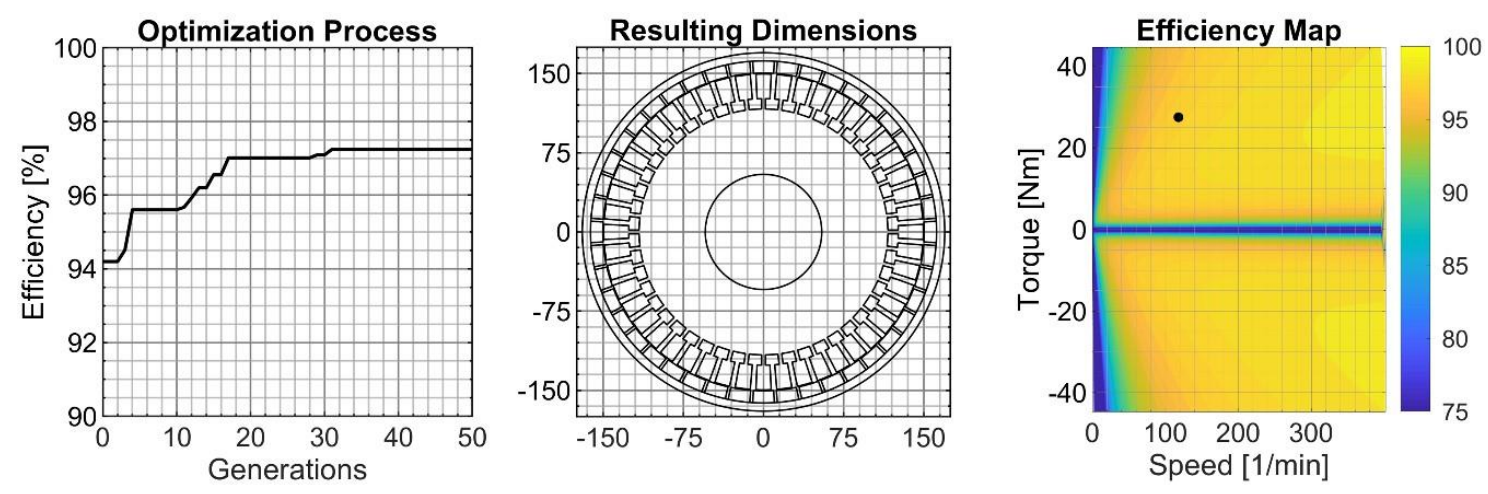

The main dimensions of the final design proposal are 339,2 $\mathrm{mm}$ for the outer diameter, $17,15 \mathrm{~mm}$ for the thickness and 0,8 $\mathrm{mm}$ for the air gap width. In the design point, the efficiency is $97,3 \%$ and the power output is $340,4 \mathrm{~W}$. The rated speed is 399,5 $1 / \mathrm{min}$ and the rated torque is $22,4 \mathrm{Nm}$, resulting in a nominal power of $936,4 \mathrm{~W}$.

\subsection{Design Point 3: $T$ and $n$ at $P_{\max }$}

In case of using torque and speed at the output power maximum, the design optimization process results in this design proposal. In Figure 6 on the left, the course of the optimization can be seen, the geometry is illustrated in the middle and on the right, the efficiency map is presented.

Figure 6. Results of the design optimization process with respect to design point 3 , indicated as a black dot in the efficiency map on the right. 


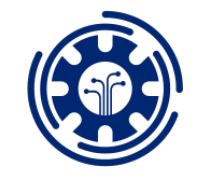

$8^{\text {th }}$ International conference on Research in Engineering, Science and Technology Paris, France
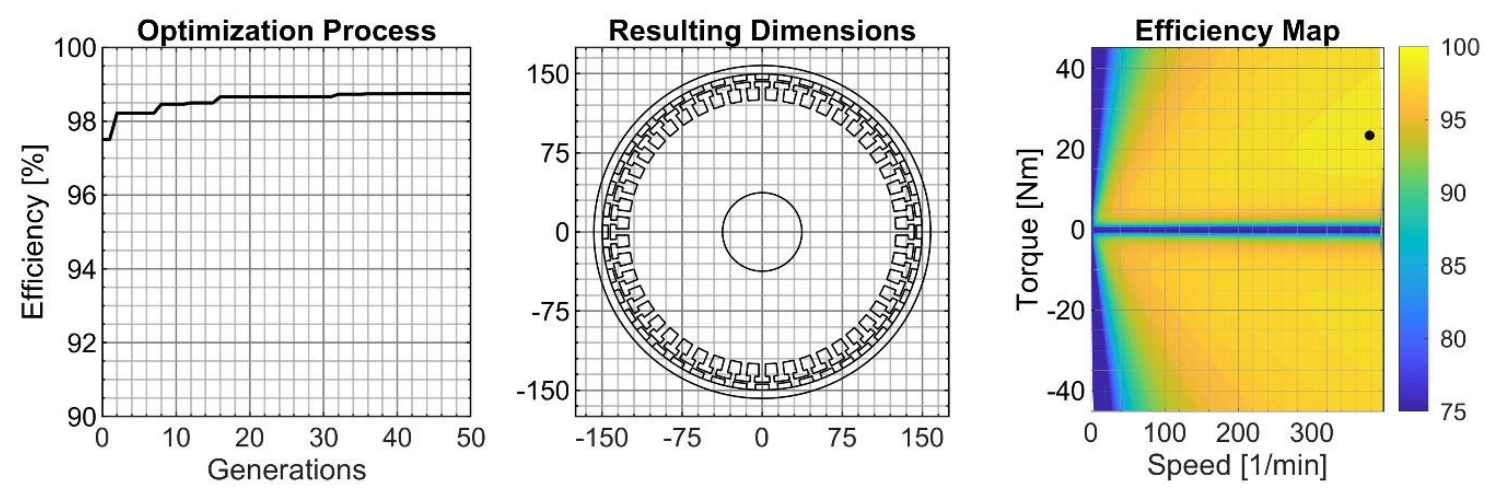

The presented design proposal of the PMSM features an outer diameter of

$315,4 \mathrm{~mm}$, a thickness of $30,1 \mathrm{~mm}$ and an air gap width of 1,9 $\mathrm{mm}$. The design point is characterized by an output power of $925 \mathrm{~W}$ and an efficiency of 98,8\%. Concerning the rated torque and speed, this leads to values of $22,6 \mathrm{Nm}$ and $395,11 / \mathrm{min}$ resulting in a nominal power of $936,4 \mathrm{~W}$.

\subsection{Working Cycle}

The last design optimization is carried out with respect not to a single design point but to the whole working cycle of the PMSM. In Figure 7, the course of the optimization, the geometry and the efficiency map can be seen.

Figure 7. Results of the design optimization process with respect to the working cycle, which is depicted as the black line in the efficiency map on the right.
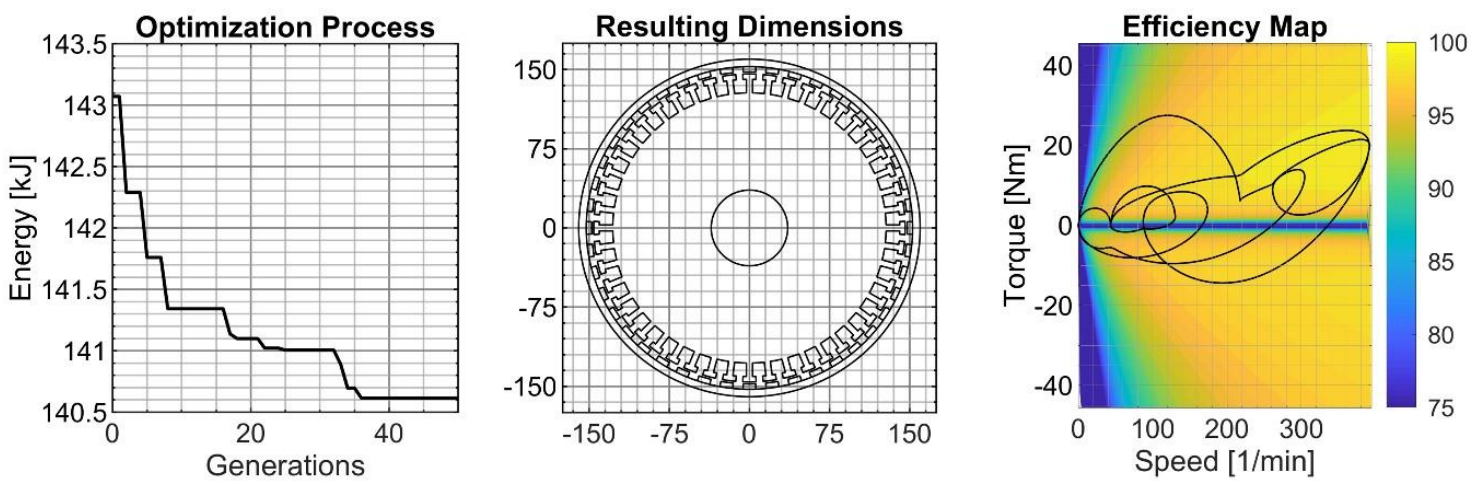

The main dimensions of the last design proposal resulted in an outer diameter of $319,6 \mathrm{~mm}$, a thickness of $28,7 \mathrm{~mm}$ and an air gap width of $1,9 \mathrm{~mm}$. A specific efficiency cannot be provided here, since the computation of the overall energy required in performing the working cycle was the objective. For the whole working cycle an energy of $140,6 \mathrm{~kJ}$ is required. The rated torque and speed are $22,8 \mathrm{Nm}$ and $392,11 / \mathrm{min}$, resulting in a nominal power of $936,8 \mathrm{~W}$. 


\subsection{Comparison}

In this case study, the objective was to optimize the design of a PMSM for different design points respectively a working cycle. By comparing the different design proposals with respect to the design points, the efficiency and energy values given in Table 2 arise.

Considering the efficiency values in the three different design points, it is obvious which design proposals offered the highest values. As expected, the design proposals offered the highest efficiency in the respective design points, which were used in the design optimization. But more important than the efficiency in a specific design point is the overall energy the motor needs to conduct the working cycle. In this case, the last design proposal, which was designed with respect to the operational cycle needs the least amount of energy.

Table 2. Efficiency and energy values of the design proposals for the design points and the working cycle.

\begin{tabular}{lcccc}
\hline & Design Point 1 & Design Point 2 & Design Point 3 & Working Cycle \\
\hline Optimization: Design Point 1 & $98,3 \%$ & $97,0 \%$ & $98,7 \%$ & $140,8 \mathrm{~kJ}$ \\
Optimization: Design Point 2 & $98,0 \%$ & $97,3 \%$ & $98,6 \%$ & $141,1 \mathrm{~kJ}$ \\
Optimization: Design Point 3 & $98,1 \%$ & $96,5 \%$ & $98,8 \%$ & $141,0 \mathrm{~kJ}$ \\
Optimization: Working Cycle & $98,3 \%$ & $95,8 \%$ & $98,8 \%$ & $140,6 \mathrm{~kJ}$ \\
\hline
\end{tabular}

Especially the comparison of the proposals for design point 1 and the working cycle is revealing. Although the first design proposal offers higher or equal efficiency values in two of three design points, the overall required energy is higher than for the last design proposal. Presumably the efficiency of the design proposal for design point 1 is lower in areas which are not covered by the design points compared to the design proposal which was designed with respect to the working cycle. This leads in total to a higher amount of energy required.

\section{Conclusion}

This paper was intended to emphasize the importance of operational cycles for the design of energy-efficient electric motors. Based on a literature survey, recent design methods were briefly presented. It turned out that methods, which include the operational cycle partly into the design process are rare and they condense the working cycle into a set of design points. To show the importance of including the operational cycle into the design process, an electric motor was designed in order to function in an electric scooter. The boundary conditions, which had to be fulfilled by the final design proposal were mainly the maximum diameter of the rotor and the thickness. Additionally, the motor should be able to perform a working cycle, derived from a desired driving cycle of the electric scooter. To evaluate, how different design points affect the resulting motor 
proposals in terms of efficiency and required energy, four design optimizations were conducted. The first free were done using the efficiency in specific design points as the objective. For the fourth design optimization, the energy required to conduct the working cycle was used as the objective. The first three motor designs had high efficiency values in their respective design points compared to the other proposals. Concerning the required energy for the working cycle, the fourth design proposal was superior to the other. This motor design had not the best efficiency values in every design point, but the overall energy was the lowest. Therefore, it can be assumed that this design proposal has higher efficiency values where it is necessary due to the working cycle and lower ones where it is unimportant. In this particular design optimization, the admissible diameter of the motor was so large that the different efficiency and energy values were quite close to each other. For stricter boundary conditions or multi-objective optimizations, where for instance the weight or the cost are considered additionally, the differences would be more prominent. It can be stated that the design optimization with regard to a single design point will not necessarily lead to an energy-efficient motor design concerning a working cycle. To achieve a better motor design in terms of energy requirement, the whole working cycle has to be considered in the design optimization.

\section{References}

Crozier, R., Mueller, M. (2016). A new MATLAB and octave interface to a popular magnetics finite element code. 2016 XXII International Conference on Electrical

Machines (ICEM), Lausanne, Switzerland, 04.09.2016 - 07.09.2016, 1251-1256. doi: 10.1109/ICELMACH.2016.7732685

Fatemi, A., Demerdash, N. A. O., Nehl, T. W., Ionel, D. M. (2016). Large-Scale Design Optimization of PM Machines Over a Target Operating Cycle. IEEE Transactions on Industry Applications, 52(5), 3772-3782. doi: 10.1109/TIA.2016.2563383

Haifeng, Z., Zhi, D. and Jinghua, Z. (2017). Optimization design and analysis of permanent magnet synchronous motor based on VC. 20th International Conference on Electrical Machines and Systems (ICEMS), Sydney, Australia, 11.08.2017 14.08.2017, 1-4. doi: 10.1109/ICEMS.2017.8055957

Jung, D.-H., Lee, J.-K., Kim, J.-Y., Jang, I. S., Lee, J. and Lee, H.-J. (2018). Design method of an ultra-high speed PM Motor/Generator for Electric-Turbo Compounding System. IEEE Transactions on Applied Superconductivity, 28(3), 1-4. doi: 10.1109/ TASC.2018.2790917

Khelifa, M., Mordjaoui, M. and Medoued, A. (2017). An inverse problem methodology for design and optimization of an interior permanent magnetic BLDC motor. 
International Journal of Hydrogen Energy, 42(28), 17733-17740. doi: 10.1016/ j.ijhydene.2017.02.017

Li, Q., Fan, T., Wen, X., Li, Y., Wang, Z. and Guo, J. (2017). Design optimization of interior permanent magnet synchronous machines for traction application over a given driving cycle. IECON 2017 - 43rd Annual Conference of the IEEE Industrial Electronics Society, Beijing, China, 29.10.2017 - 01.11.2017, 1900-1904. doi: 10.1109/ IECON.2017.8216321

Meeker, D. (2018): Finite Element Method Magnetics 4.2. Available at: www.femm.info Müller, G., Vogt, K. and Ponick, B. (2008). Berechnung elektrischer Maschinen, WileyVCH, Weinheim, Germany.

Pyrhönen, J., Jokinen, T., Hrabovcová, V. and Niemelä, H. (2010). Design of rotating electrical machines, Wiley, Chichester, UK.

van Werkhoven, M. and Advani, A. (2017). Accelerating the Global Adoption of Energy-

Efficient Electric Motors and Motor Systems. Available at: www.united4efficiency.org/wp-content/uploads/2017/09/U4E-MotorGuide201709-Final.pdf (accessed 9 September 2018)

Waide, P. and Brunner, C. U. (2011). Energy-Efficiency Policy Opportunities for Electric Motor-Driven Systems. Available at: www.iea.org/publications/freepublications /publication/EE_for_Electric Systems.pdf (accessed 9 September 2018)

Wu, D., Fei, W. and Luk, P. C.-K. (2015). Design optimizations of outer-rotor permanent magnet synchronous machines with fractional-slot and concentrated-winding configurations in lightweight electric vehicles. 2015 6th International Conference on Power Electronics Systems and Applications (PESA), Hong Kong, Hong Kong, 15.12.2015 - 17.12.2015, 1-8. doi: 10.1109/PESA.2015.7398956

Zhu, W., Yu, Z., Zhou, W., Shi, J. and Yin, Y. (2017). Design of permanent magnet synchronous motor based on genetic algorithm in unmanned ground vehicles. 2017 IEEE International Conference on Unmanned Systems (ICUS), Beijing, China, 27.10.2017 - 29.10.2017, 572-575. doi: 10.1109/ICUS.2017.8278410

Zuopeng, D., Bing, Z. and Dejun, Y. (2017). A design of fractional-slot concentrated winding IPM synchronous motor for electric vehicles. IECON 2017 - 43rd Annual Conference of the IEEE Industrial Electronics Society, Beijing, China, 29.10.2017 01.11.2017, 1668-1673. doi: 10.1109/IECON.2017.8216283 